\title{
GESTIÓN PARA EL DESARROLLO LOCAL EN TENANCINGO, MÉXICO
}

\author{
Alicia Yaneth Vásquez González \\ Licenciada en Turismo \\ Maestra en Agroindustria Rural, Desarrollo Territorial y Turismo Agroalimentario \\ Doctora en Ciencias Agropecuarias y Recursos Naturales de la Universidad Autónoma \\ del Estado de México (UAEMÉX). Miembro del Sistema Nacional de Investigadores \\ del Consejo Nacional de Ciencia y Tecnología. \\ yaly2003a@hotmail.com.
}

\section{Resumen}

En los últimos años, hay cambios tanto en los procesos económicos, como el desarrollo de la gestión pública. El primero perjudica la venta de los pequeños productores, y el segundo ayuda a las personas a participar en las decisiones de los proyectos. Porque la gestión pública anterior era centralizadora y no resolvía los problemas de los ciudadanos. Es así, que hubo una apertura al desarrollo local, donde el sector, público, privado y social se deben organizar para resolver diferentes problemas, entre ellos los económicos y ambientales. Para el desarrollo de la investigación se utilizó el método histórico y la Metodología de Sistemas agroalimentarios localizados (SIAL). El Objetivo, es describir las gestiones realizadas en el Municipio de Tenancingo, para el desarrollo local, aprovechando los recursos naturales y culturales. Existen diferentes estrategias de promoción que se han implementado en el municipio de Tenancingo, para lograr el desarrollo local; sin embargo, ninguna será efectiva mientras no exista una cooperación entre los tres sectores, para el mantenimiento, cuidado y uso de los atractivos.

Palabras clave: gestión pública, procesos económicos, recursos culturales y naturales, atractivos. 


\title{
MANAGEMENT FOR LOCAL DEVELOPMENT IN TENANCINGO, MEXICO ${ }^{1}$
}

\author{
Alicia Yaneth Vásquez González. \\ Doctora en Ciencias Agropecuarias y Recursos Naturales de la Universidad Autónoma \\ del Estado de México (UAEMÉX). Miembro del Sistema Nacional de Investigadores \\ del Consejo Nacional de Ciencia y Tecnología. Profesor de asignatura de la UAEMÉX. \\ yaly2003a@hotmail.com.
}

\begin{abstract}
In recent years, there have been changes in both economic processes and the development of public management. The first one harms the sale of the small producers, and the second one helps the people to participate in the decisions of the projects. Because the previous public management was centralizer and did not solve the problems of the citizens. It's like that, that there was an opening to local development, where the sectors, public, private and social must be organized to solve different problems, including economic and environmental. For the development of the research, the historical method and the Localized Agri-Food Systems Methodology (SIAL) were used. The objective is to describe the managements made in the Municipality of Tenancingo, for local development, taking advantage of natural and cultural resources. There are different promotion strategies that have been implemented in the municipality of Tenancingo, to achieve local development; however, none will be effective as long as there is no cooperation between the three sectors, for the maintenance, care and use of the attractives.
\end{abstract}

Keywords: public management, economic processes, cultural and natural resources, attractive.

1 Parte de la información fue extraída de mi Tesis de Licenciatura "Análisis de las Perspectivas del Desarrollo del Turismo Local en el Municipio de Tenancingo" y de Maestría "La producción artesanal de pan en "San Miguel Tecomatlán", como un recurso para proponer un proyecto de agroturismo". 


\section{Introducción}

A nivel internacional los procesos económicos están cambiando y van marginando a las zonas rurales (Flores, et al., 2014). Cada día siguen aumentando las tendencias de liberación del comercio mundial, y la libre competencia, perjudicando a los comerciantes internos (Flores, et al., 2014), entre ellos los municipios. Los cambios en la economía global, han hecho que las localidades jueguen un papel importante en el desarrollo económico de los municipios (Pérez, S/A).

Sin embargo, la gestión pública centralizada presenta un sin número de problemas y críticas, porque en vez de solucionar problemas de la ciudadanía se resolvían problemas de cada uno de los servidores públicos (Aparicio, 2009); por ello, se inició la apertura a un nuevo modelo de gestión encaminado al desarrollo local (Ornés, 2009), donde exista eficacia y eficiencia, la participación de la ciudadanía, la transparencia, y el mejoramiento de la administración de los recursos (Aparicio, 2009), que responda a las demandas de los ciudadanos. Es así, que se pretende que la nueva gestión pública beneficie la economía de los habitantes.

Para la nueva gestión pública se .requiere que los sectores público, privado y social se involucren en diferentes actividades, que beneficien a todos. Y también que exista un cambio en la operatividad; es así, que se ha intentado que los municipios se independicen y que se consiga un desarrollo económico local. En este caso se pretende dicho desarrollo a través del turismo. El turismo en los últimos años constituye el tercer lugar en la economía mundial; es así, que la actividad ha alcanzado enormes relaciones económicas y socio-culturales (Venturini, 2015).

México ocupa el octavo lugar a nivel internacional y el décimo cuarto en ingresos de divisas según datos de la Organización Mundial de Turismo, en el ranking 2016 y fue el segundo en América Latina en recibir a turistas, después de Estados Unidos (Sectur, 2018). Es así, que el turismo forma parte del desarrollo económico de los lugares. Y entonces puede ser un factor importante para mejorar las economías de los municipios; pero sin dejar de lado el cuidado del medio ambiente.

Tenancingo, Estado de México, tiene una serie de recursos naturales y culturales, que pueden ser aprovechados para atraer turistas de forma racional y obtener un beneficio económico. La participación del gobierno, de los empresarios y de los pobladores, es importante para que se realicen proyectos de promoción y mantenimiento de los atractivos, y así sea un detonante del desarrollo local. 


\section{Metodología}

Para el desarrollo de la investigación se utilizó el método histórico, el cual es aquel en el que captamos los contenidos descriptivos ${ }^{2}$ y explicativos del pasado de las sociedades, de los grupos que las constituyen, de sus instituciones y de cualquier fenómeno social concreto. En esta parte se revisó la historia de Tenancingo, para conocer cómo se ha ido desarrollando en turismo en el lugar y los cambios que se han presentado. Asimismo, se elaboró un diagnóstico para dar inicio a un proceso de planeación turística. Se obtuvieron diferentes datos de los tres sectores que ayudan a proyectar un desarrollo local.

Se realizaron entrevistas (guía de entrevista) a los dueños de los servicios turísticos, al presidente, y a la coordinación de turismo. Se realizaron 116 cuestionarios para los turistas, de un total de 729 . En dicha muestra se consideró la de poblaciones finitas. También se aplicaron cédulas de identificación y evaluación a los diferentes recursos naturales y culturales, para saber su estado de conservación.

También se usó la Metodología Sistemas agroalimentarios localizados (SIAL). El enfoque es una invitación que se les propone a los pequeños productores rurales, para que puedan resistir a los cambios que se han dado a nivel global, se les presentan diferentes modelos de desarrollo agroalimentarios, donde se valoran los recursos locales (Muchnik, 2006).

Para la obtención de la información se realizó un diagnóstico (territorio, actores, producto), diálogo para la activación (FODA), y acompañamiento del proceso con sesiones participativas comunitarias con productores, la activación y puesta en marcha (Boucher, et al., 2011). También la observación directa, las entrevistas estructuradas y no estructuradas, y de forma alterna se aplicó un cuestionario a visitantes. Dicho cuestionario es para conocer si es factible la realización de una expo-feria en la cabecera municipal, por medio de la muestra no probabilística. El método histórico y el SIAL, sirvieron de base para realizar propuestas que sugieren la participación de la población para su desarrollo.

\section{Localización del área de estudio}

Tenancingo se considera una ciudad típica, con clima templado y consta de una variada y nativa vegetación, tiene construcciones típicas; sin embargo, se encuentran deterioradas como es el palacio municipal (Gaceta municipal, 2013). Es así, que las construcciones han ido cambiando a través de los años y se va perdiendo el diseño homogéneo propio del contexto histórico al que pertenece el municipio.

2 Busca especificar propiedades, características y rasgos importantes de cualquier fenómeno que se analice, describe tendencias de un grupo o población (Hernández, et al., 2010). 
Dicho municipio es parte del Estado de México, y se encuentra al sur del Estado. Limita al norte con Tenango del Valle y Joquicingo. Al sur con Zumpahuacan, al poniente con Villa Guerrero y al oriente con Malinalco. Se distingue por ser un centro de intercambio comercial entre varios municipios como: Malinalco, Zumpahuacan, Villa Guerrero, Coatepec harinas y Tenango del Valle. Su extensión territorial es de $163.59 \mathrm{~km} 2$ (INEGI, 2013).

Foto: Tenancingo
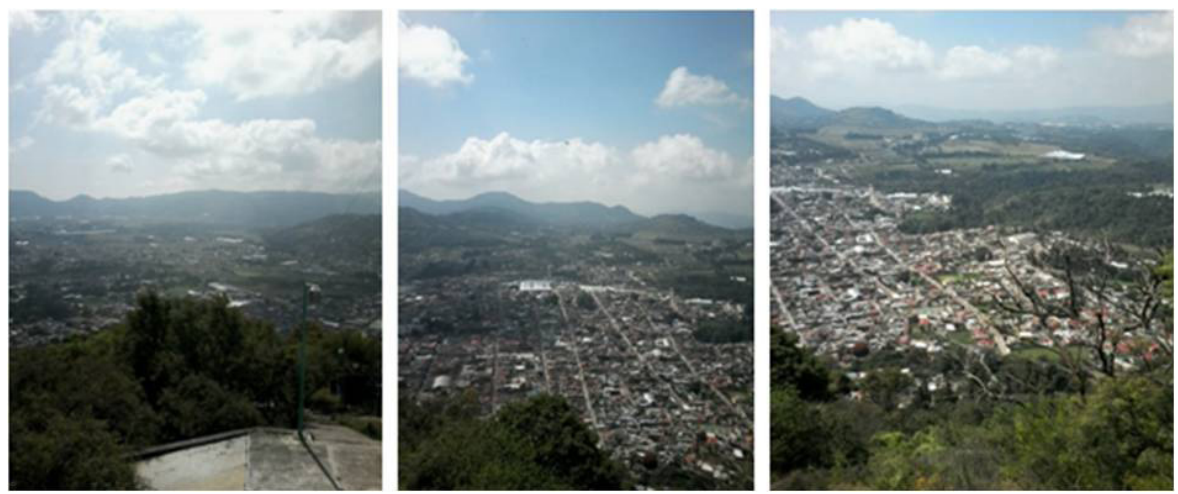

Fuente: Yaneth Vásquez, 2016.

El municipio tiene 90, 946 habitantes, de los cuales 44 mil 239 son hombres y 46 mil 707 son mujeres; de los cuales el $53.91 \%$ es urbana y el $46.09 \%$ se clasifica como rural (Gaceta municipal, 2013). En general, según datos obtenidos de la Gaceta municipal al municipio se le considera como una localidad con todos los servicios públicos como drenaje, agua potable, entre otros.

Tenancingo se ha incorporado al corredor turístico Distrito Federal-Toluca-TenancigoIxtapa de la Sal-Taxco, Guerrero, cuya estrategia de desarrollo está orientada a abatir el aislamiento del municipio, y con dicha inserción se pretende impulsar las actividades propias de la región (Gaceta municipal 2013). Considerando la llegada de turistas para mejorar la economía del municipio y se pueda generar un desarrollo local.

\section{Desarrollo local}

"La planificación de desarrollo regional solía estar muy apegada a los planes maestros y al desarrollo desde arriba. Actualmente los gobiernos regionales tratarán de crear un entorno adecuado de las actividades económicas" (Ortega, 1994:99). Y así, considerar los problemas que se presentan en las comunidades, para formular planes y programas, que lleven a resolver los problemas de la ciudadanía y no de unos cuantos. 
"En materia de regulación del uso del suelo y administración urbana se ha fomentado la adecuación de la legislación urbana a nivel local, la modernización de los servicios públicos en la ciudad y la definición de programas de ordenación de los centros de población" (Ortega, 1994:20). Con dicha regulación se pretende el buen funcionamiento de los municipios, que llevé a un avance económico, para llegar a un desarrollo local.

\section{El Desarrollo Económico Local puede definirse como:}

"Aquel proceso reactivador de la economía y dinamizador de la sociedad local que mediante el aprovechamiento de los recursos endógenos existentes en una determinada zona o espacio físico es capaz de estimular fomentar su crecimiento económico, crear Empleo, Renta y Riqueza y sobre todo mejorar la Calidad de Vida y el Bienestar Social de la Comunidad Local" (Pérez, S/A:03).

"El Desarrollo Local es un proceso que se construye diferenciadamente en cada país según las distintas articulaciones que se producen entre las dimensiones territoriales, la historia, las estructuras y la acción diferenciada de los actores" (Cárdenas, 2002:60). El concepto, intenta ubicar al ser humano y a los intereses colectivos como punto central, fomentando y desarrollando las actividades de los individuos (Juárez, 2013).

El desarrollo participativo se propone, para que el proyecto en cuestión sirva para empoderar a los pobladores, en el sentido de analizar y solucionar sus propios problemas (Vázquez, 2003). Es un empoderamiento que tiene la población ante el gobierno ya sea local, regional o nacional. Pero es un reto complicado, porque hay que hacer entender a los actores sobre la necesidad de establecer una nueva forma de trabajo (Velasco, 2009).

Sin embargo, se dice que muchos de los proyectos no empoderan a las poblaciones, que únicamente se obtiene información sobre ellas, y el control sigue siendo limitado (Vázquez, 2003). Por ello, las comunidades deben de organizarse para ser parte de los proyectos y obtener beneficios de cada uno de ellos. Y así los actores deben comprender que los nuevos proyectos son de responsabilidad compartida (Velasco, 2009), no exclusivos del sector público o privado, y que las poblaciones pueden recibir a cambio servicios como: una clínica de salud, una escuela, entre otros.

El desarrollo local se fortalece a medida que avanza el siglo XXI, y se buscan alternativas económicas para que fortalezcan a las comunidades rurales (Flores, et al., 2014). Es necesario mejorar los componentes del entorno locales como infraestructura básica (económica o técnica, sociales, ambientales), la cooperación entre empresas, la utilización sostenible de los recursos naturales (Alburquerque, S/A), entre otros. 
En los últimos años se han desarrollado varios modelos que pretenden incluir la participación del sector público, social y privado, para lograr el desarrollo local. Para esta investigación se consideró el Modelo de la Comisión Económica para América Latina y el Caribe (CEPAL), porque entre sus puntos importantes describe la participación conjunta de los tres sectores. Sus puntos son:

Primero. Debe haber una buena organización entre la comunidad, gobierno local y empresas.

Segundo. El gobierno local, la comunidad y los empresarios deben tener diferentes instituciones que ayuden al buen funcionamiento del convenio y cada uno respete la parte que le corresponde realizar, hay que considerar la estructura política-social.

Tercero. Se deben considerar los proyectos de inversión.

Cuarto. Se deben considerar los convenios, porque así se imponen las reglas a los participantes, y para que cada uno realice la parte que le corresponde de trabajo.

Es así, que el desarrollo local es un enfoque que quiere impulsar el desarrollo endógeno, la auto-organización y el bienestar social, lo que implica la participación de todos ya sea individual o colectiva (Juárez, 2013), entonces debe aumentar la participación social, y el Estado debe ejercer una intervención estratégica con los demás actores sociales, en la promoción y sostenimiento del desarrollo (Cárdenas, 2002).

El desarrollo local pretende que exista la participación de los tres sectores: social, público y privado, para cualquier gestión. Es así que la población y las organizaciones locales, pueden formular sus deseos y estos se pueden ejecutar. Los deseos pueden ser de diferentes cosas por ejemplo: infraestructura, educación, capacitación a nivel local, entre otros, ya que el gobierno tiene la obligación de implementar este tipo de servicios, porque su labor es dar atención.

"El impulso a las ciudades medias se ha consolidado a través de la construcción de obras de infraestructura y dotación de servicios urbanos, a aquéllas con mayor potencial de desarrollo, procurando que la inversión pública tenga un efecto multiplicador sobre la actividad económica de los sistemas urbanos regionales" (Ortega,1994:20).

Para lograr lo anterior, se previó la realización de acciones orientadas a apoyar la generación de un mayor ahorro de los gobiernos estatales y municipales, en forma tal que puedan satisfacer sus necesidades de inversión y el otorgamiento de servicios básicos a la población. Al respecto, se pueden integrar sistemas de análisis para el ahorro y para el 
cobro, considerando que se obtienen una serie de pagos en el municipio que pueden ser potencializados.

Entonces, se pretende integrar al turismo como eje principal, para el desarrollo local. El turismo local, es cuando se promocionan los recursos endógenos que existen en cierta zona, para que la gente los visite (Rodríguez, 2015), considerando el cuidado del medio ambiente. Es mostrar una serie de atractivos de un lugar, para provocar que llegue la gente y deje dinero. Y que al existir la unión de acciones, entre los tres sectores: social, público y privado, pueda generar el cuidado y la promoción de los recursos culturales y naturales, y así atraer el turismo.

La OMT (Organización Mundial del Turismo), (2018) llama a los diferentes países a considerar al turismo, como un punto importante, para reducir la pobreza; también publica que se debe concientizar sobre lo viable que es el sector para el desarrollo. Es así, que en México se puede aprovechar el potencial turístico que se tiene, y generar mayor derrama económica en el país.

Además se considera al turismo como fuente de trabajo, que puede incrementar la operación de pequeñas y medianas empresas, sin dejar de lado el cuidado de la riqueza natural y cultural de los lugares (PND, 2013-2018). Así como integrar a las poblaciones que reciben a turistas, para que obtengan un beneficio económico y con ello mejores condiciones de vida.

Para ello, todas las políticas de desarrollo turístico, deben ser enfocadas a reducir la pobreza y a incluir a la población (PND, 2013-2018). "Una evidencia al respecto es que $87 \%$ de la población en municipios turísticos en nuestro país tiene un nivel de marginación "muy bajo" de acuerdo con el CONEVAL, mientras que la cifra equivalente en los municipios no turísticos es de 9 por ciento" (PND, 2013-2018:1). Tenancingo presenta un nivel de marginación "media", con un rezago social "bajo" (Estadística, 2013), y no es considerado un lugar turístico como lo es Ixtapan de la Sal (INEGI, 2017).

Sin embargo, los lugares turísticos son importantes, porque se tienen ingresos adicionales que pueden apoyar a las familias tenancinguenses, sobre todo si existe la participación de los tres sectores para su desarrollo; es así, que hay que aprovechar el crecimiento de visitantes y promover el municipio. En Tenancingo se pueden integrar estrategias de promoción, para que lleguen más visitantes nacionales e internacionales. 


\section{Gestión para el desarrollo local}

En la actualidad la gestión pública lleva a fortalecer al municipio, en la equidad asociada con un mejor acceso al poder político y al empleo productivo, permite el encuentro de los ciudadanos con sus gobernantes y la apertura de la mediana y pequeña empresa (Cárdenas, 2002). El turismo es una actividad socio-económica nacional, que es importante a nivel municipal, porque se tienen potencialidades, donde se puede desarrollar la gestión (Rodríguez, 2015).

Además es un instrumento de desarrollo local, que permite una distribución equitativa de los beneficios que se obtienen, pueden ser económicos, sociales y/o culturales, que se debe reflejar en la mejora de la educación, en la creación de empleos y por tanto un mejor ingreso económico (Martínez, y Escalona, 2012). Sin dejar de lado el cuidado del medio ambiente, como lo menciona el Secretario General de la Organización Mundial del Turismo.

El secretario General de la OMT dice que el año 2017, es el Año Internacional del Turismo Sostenible para el Desarrollo, y que debemos reflexionar sobre cómo gestionar el turismo de una manera responsable y sostenible, que se necesitan extender los beneficios sociales y económicos del turismo, pero reduciendo los impactos negativos en las comunidades receptoras, para ello todos debemos participar y dar nuestros máximos esfuerzos (OMT, 2017). Es así, que hay que adoptar tácticas que permitan la participación de los tres sectores en el turismo, y en la protección del medio ambiente (Ornés, 2009).

Además en el Plan Nacional de Desarrollo (PND), 2013-2018, se hace un llamado a aprovechar el potencial turístico de México, para generar una mayor derrama económica en el país. También se reconoce que en los últimos años el turismo ha sido una palanca para el desarrollo nacional, que se han generado más empleos y se han obtenido divisas.

Lo anterior, se entrelaza con la nueva gestión pública, donde se pretende que los municipios sean autónomos y manejen sus propios programas y proyectos, que las comunidades tengan los apoyos correspondientes en la utilización de sus recursos, para aprovecharlos; ya sean los naturales y/o los culturales. En este caso el proyecto sería sobre una mayor apertura del turismo en la zona, considerando la promoción de los recursos naturales y culturales, con atractivo. Y al mismo tiempo el cuidado del medio ambiente. 


\section{Resultados}

Las gestiones que se realizan en el mundo son diferentes, porque cada zona tiene sus propias particularidades. El municipio de Tenancingo tiene recursos naturales y culturales que pueden promocionarse para que los turistas los visiten. Es así, que a través del turismo se pretende impulsar los recursos de la región, que la organización de la misma gente (políticos, empresarios y todos aquellos que integran el municipio) ayude a que se genere el desarrollo local.

Las gestiones se iniciaron en el 2001 y se ha ido trabajando a través de los años implementando, diferentes estrategias para lograr el desarrollo local. Se empezó con los empresarios para saber qué tan dispuestos estaban para trabajar en conjunto con el ayuntamiento y la comunidad, de la misma manera y al mismo tiempo se platicó con la comunidad y con el gobierno local.

Los empresarios dijeron que estaban de acuerdo en trabajar para lograr un beneficio económico, pero con la condición que el gobierno no participara, porque según lo único que quería los gobernantes es el beneficio propio. De la misma manera reaccionó la comunidad y el gobierno local, cada uno por su lado dijeron los mismo del otro. Así mismo, el gobierno local accedió a ciertas actividades, pero que no lo obligaran a dar dinero para desarrollar los trabajos.

Para lograr el desarrollo local, según el modelo CEPAL, se requiere de la participación conjunta de los tres sectores para lograr:

Primero. Una buena organización entre la comunidad, el gobierno local y las empresas. En ese sentido Tenancingo en el año 2001 carecía de organización entre los tres sectores. Es decir, cada uno trabajaba por su lado. Sin embargo, desde el 2010 se vio un avance, aunque los tres siguen pensando que el otro quiere sacar provecho; pero, quieren trabajar por un bien común, porque las personas que viven en Tenancingo, por lo general son las que tienen un negocio. Es por ello que empiezan a trabajar en conjunto, con muchas deficiencias de todos lados, pero con la intención de crecer y obtener beneficios económicos para subsistir.

Segundo. Que lo tres sectores, deben tener diferentes instituciones que ayuden al buen funcionamiento del convenio y cada uno respete la parte que le corresponde realizar, hay que considerar la estructura política-social. Tenancingo, necesita mejoras, para el desarrollo del turismo y así se pueda generar desarrollo económico; porque las instituciones funcionan a medias y los convenios no existen. 
Desde el 2010, se ha investigado sí existen asociaciones de empresarios, o no, y en conclusión se llegó a que existen varias. Dichas asociaciones presentan una serie de problemas entre sus agremiados, pero que no han sido suficientes para terminar las agrupaciones. Cada grupo ha trabajado para obtener beneficios para sus agremiados. En esta investigación se aborda la organización de los panaderos de Tecomatlán, en donde se dice que existen malos manejos en lo económico. Pero a pesar de las quejas, la organización ha logrado varios apoyos que antes no tenían. Ante las organizaciones que existen en la zona, el gobierno se ve obligado a dar beneficios, para los empresarios, organizaciones, y pueblo en general.

Tercero. Hay que considerar los proyectos de inversión. En el Plan de desarrollo municipal 2013-2015, consideran impulsar la inversión para el mantenimiento de los atractivos turísticos; así como el cuidado del medio ambiente.

Cuarto. Debe haber convenios, para que cada uno realice la parte que le corresponde de trabajo. El gobierno como los empresarios tienen desconfianza del otro, sin embargo; se dice que existen convenios entre los participantes pero no se tienen datos escritos (convenios), en lo que se refiere al mantenimiento y promoción de los atractivos turísticos.

Durante años no hubo intención del sector público y privado, de avanzar en el mantenimiento de los recursos disponibles para impulsar el turismo. Sim embargo, se siguió con la investigación, para ello se realizó un cuestionario. En donde los visitantes contestaron amablemente las preguntas. Y los resultados obtenidos fueron: el 90\% de los entrevistados, dijeron que se le diera promoción a los atractivos que se mencionaban, porque el $45 \%$ sólo conocía dos o tres atractivos de los nombrados en el cuestionario: el Cristo Rey, Convento Carmelita y Salto de Santa Ana.

Así mismo, el $88 \%$ consideraron que Tenancingo necesita infraestructura turística y más servicios, que Tenancingo era bonito y que les gustaría conocer más lugares tanto naturales como culturales en un $60 \%$. Por lo tanto, Tenancingo es un lugar que gusta a los visitantes, pero hace falta un sin número de servicios básicos que son importantes, sobre todo en los atractivos.

Tiempo después iniciaron a hacer mantenimiento y limpieza en la cabecera y en algunos recursos, y a poner señalamientos. Los recursos que se propusieron como atractivos turísticos en 2002 fueron: Parque Desierto del Carmen, Parque Hermenegildo Galeana, Salto de Santa Ana, Salto de San Simonito, Monumento a Hidalgo, Cristo Rey, Convento Carmelita, Templo de San Francisco de Asís, Capilla del Calvario, La Malinche y artesanías como: el rebozo, la madera, licores, y feria del jarro. 
Foto: Cristo Rey

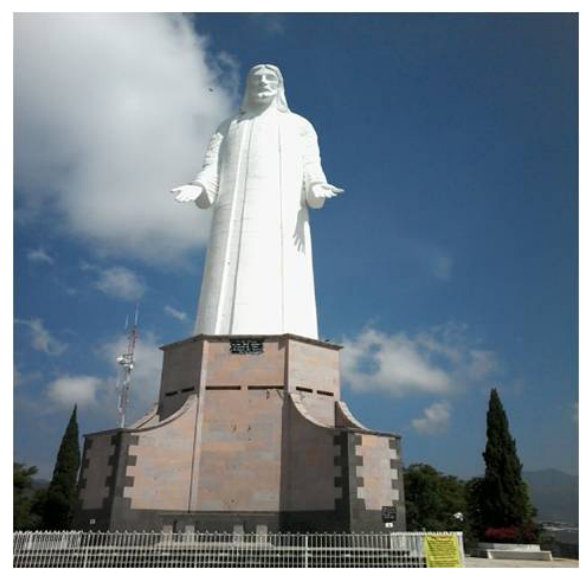

Fuente: Yaneth Vásquez, 2016

Y en el Plan Municipal Tenancingo del 2013, se muestran 10 atractivos que son: Parque Desierto del Carmen, Parque Hermenegildo Galeana, Salto de Santa Ana, Cristo Rey, Mercado municipal, mercado de la flor, El Ahuehuete, La Malinche, El Santuario del Agua, El Cerro de las Tres Marías (Gaceta, 2013). Pero, según datos recabados en campo son más recursos con los que cuenta el municipio.

Aun así, integrando únicamente los atractivos que el gobierno reconoce, deberían darles mantenimiento constante a esos lugares, situación que no existe. Los atractivos que da a conocer el gobierno local, deberían de ser conocidos y atraer a la gente. Sin embargo, los visitantes conocen uno o dos atractivos, según ellos es por falta de información.

Existe un módulo de información turística, se encuentra enfrente del Palacio Municipal en el jardín central, debajo del kiosco. Los trípticos presenta varios recursos naturales y culturales, varios de ellos se dieron a conocer desde hace varios años. El problema es que los visitantes no tienen idea de dónde buscar la información. Hace varios años el módulo permanecía cerrado todo el tiempo, en la actualidad no.

Sin embargo, los atractivos que da a conocer el gobierno local carecen de mantenimiento, de seguridad, de limpieza, y de información nacional e internacional. La información que existe de los recursos es en trípticos, y falta promocionarlos en otros medios de comunicación. Entonces se propone que sean dados a conocer en la televisión, radio e internet. En el Plan de Desarrollo Municipal, 2013-2015, se tiene previsto la promoción y su mantenimiento. Pero, en la realidad lo anterior se realiza a medias. 
En general, tanto los atractivos mencionados por el gobierno municipal, como los demás recursos que pueden ser aprovechados para el turismo, les falta mantenimiento y promoción.

Sin embargo, hay que mencionar que el gobierno local en el periodo pasado 2013-2015 y el actual 2016-2018, han realizado diferentes proyectos de infraestructura en diferentes lugares del municipio, y en esos trabajos ha integrado a personas de las poblaciones que integran Tenancingo, para trabajar en dichos arreglos y les pagan. Anteriormente el gobierno llevaba gente de fuera para realizar dichos trabajos y muchas veces no los terminaban.

Los empleos son temporales, pero han apoyado a la economía de las personas que se han integrado a dichos proyectos. Es así que se puede apreciar un cambio entre los trabajos pasados y los de ahora, pero aún existen problemas. Por ejemplo: los trabajadores son seleccionados por el delegado o por los encargados de la obra, y se dice que escogen a sus familiares o, a las personas allegadas a los políticos locales.

En campo se puede visualizar que la gente que participa, es la que tiene tiempo y quiere trabajar, o sea que no labora en otro lado. También se visualiza que los empleos son expuestos a los delegados de cada población, o a los encargados de la obra de los lugares, y ellos lo distribuyen para la gente que vive cerca de donde se van a realizar los proyectos. Pero efectivamente, se observa que los puestos son para la gente cercana a los representantes.

Es así, que en general los proyectos de mantenimiento en diferentes lugares del 2002 - al 2017 han tenido cambios, por un lado en integrar a la gente de los lugares donde se hacen los arreglos, por otro en considerar a los delegados de cada población para que ellos busques trabajadores de la zona.

Los arreglos de mantenimiento de calles, la limpieza, y los señalamientos, han sido importantes para la imagen del municipio, y se pueden considerar para la atracción de los turistas; pero los atractivos y los recursos potenciales en específico siguen careciendo de mantenimiento y promoción. También falta dar información a los visitantes, de los atractivos y de los recursos potenciales. 
Foto: Centro de Tenancingo

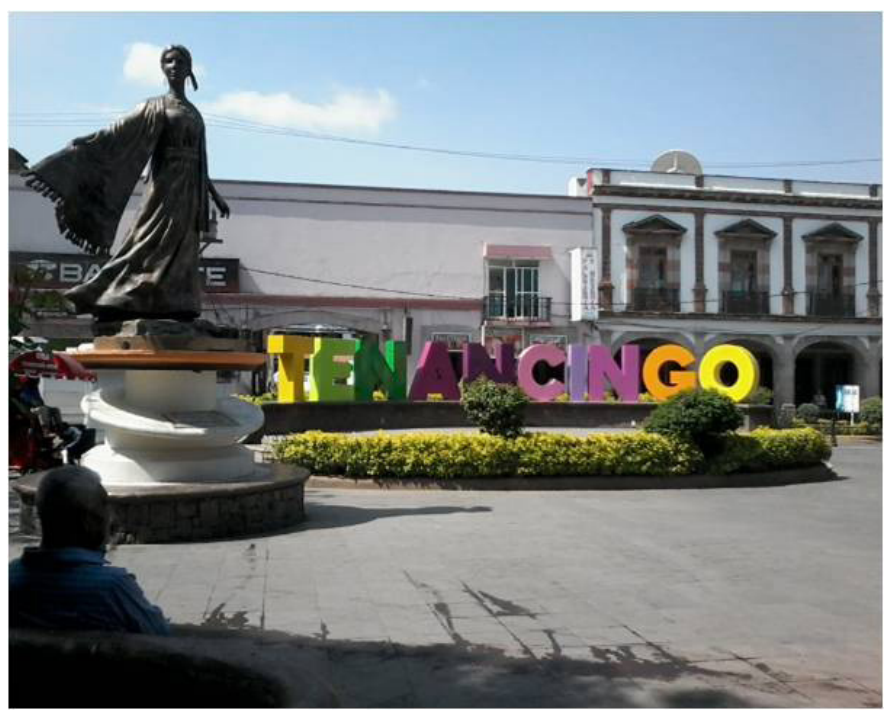

Fuente: Yaneth Vásquez, 2018

En Tenancingo, durante muchos años se ha dicho que se pretende incentivar al sector turismo; sin embargo, no se aprovechan los recursos disponibles, que se tienen para este rubro. Más bien se aprovechan para otros sectores, como es el caso de la siembra de flor.

Aunque, se han realizado proyectos para promocionar atractivos como: la feria del obispo en 2004, así como señalizaciones de algunos recursos (las señalizaciones se han puesto en lugares estratégicos de la cabecera municipal). Pero se necesitan más esfuerzos por parte del gobierno local, población y prestadores de servicios, para que en Tenancingo lleguen turistas. Porque el municipio es catalogado como zona de intercambio comercial, pero no como un municipio con atractivos naturales y culturales. 
Fotos: Tríptico Turístico de los Atractivos de Tenancingo

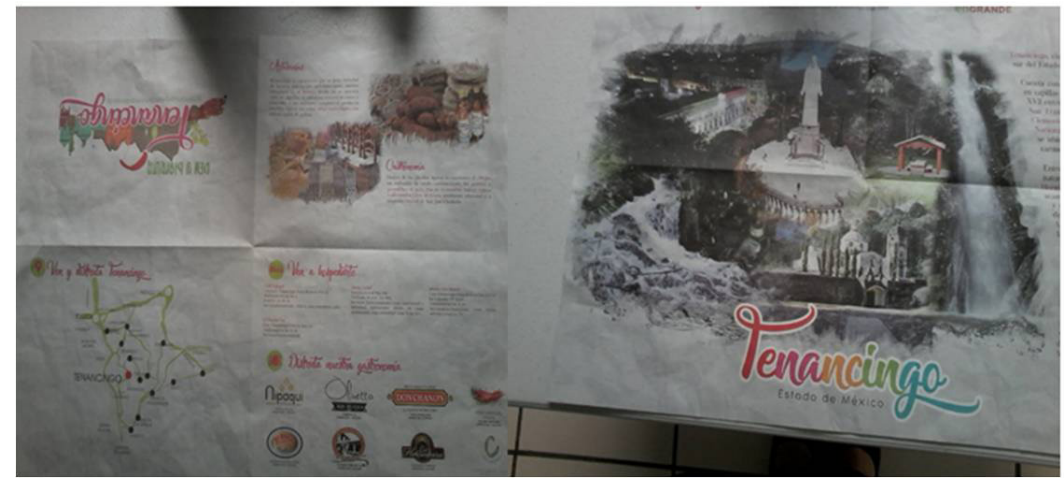

En el sentido de dar promoción a los recursos disponibles, en el 2012 se propuso un expoferia para que los artesanos del pan de Tecomatlán, tuvieran otra opción de venta. La cual se dio a conocer de manera particular para obtener el apoyo, debido a que la propuesta del 2002, no tuvo la aceptación esperada. Se piensa que es porque se pidió la promoción para varios recursos, y no había apoyo para todos.

Es por ello, que para el 2012 se pide para un solo recurso, pero aunque se gestione uno, la intención de la expo-feria es promocionar el conjunto de atractivos disponibles en Tenancingo. Y así atraer a los visitantes en diferentes ocasiones, para que conozcan un sin número de recursos naturales y culturales.

Para ello se tuvo que realizar un diagnóstico del lugar donde se elabora el pan, pero considerando el centro de Tenancingo, porque en la cabecera municipal se pretende realizar la expo-feria del pan. Para la elaboración de la propuesta, en primer lugar se consideraron a los artesanos del pan, para que eligieran que querían realizar para aumentar las ventas de su producto. Después se consultó al gobierno local, para saber su opinión. Y al final a los visitantes, para saber si les gustaría presenciar una expo-feria.

El proyecto se realizó y se entregó al gobierno local; sin embargo, no se ha avanzado en el desarrollo de la expo-feria. Aunque, se logró que los consideraran y les dieran más lugares de venta en la zona, y en temporada de muertos se realiza la venta del pan de Tecomatlán, junto con otros tipos de panes que se realizan en Tenancingo; así como el complemento de productos que se utilizan en dicha temporada. Pero se sigue pensando en realizar la expo-feria del pan de Tecomatlán. 
Tenancingo ya cuenta con varias ferias como es el caso de la feria del jarro, y la del obispo. La última que inició en 2004, con la aprobación del presidente municipal, Licenciado Ángel Luz Lugo Nava y fue para difundir el turismo en sus diversas ramas; principalmente en la rica gama gastronómica que ésta ciudad ofrece (Varela, 2011). Es así que dicha feria promueve parte de los recursos que existen en Tenancingo.

Por lo tanto, el municipio cuanta con muchos recursos tanto naturales como culturales, pero se necesita mantenimiento, limpieza, promoción, etcétera, situación que existe a medias; es decir, no se les ha dado la prioridad que se merecen. Aunque existe la idea de implementar infraestructura en los diferentes atractivos. Por ejemplo, se pretende la construcción de gradas, arreglo de plazas cívicas y de calles.

También en sus líneas de acción pretenden promover los atractivos, implementar programas de promoción y capacitación, llevar a cabo ferias y exposiciones de productos artesanales (Gaceta, 2013); aunque, en la realidad no se visualiza. Es así que existe una contradicción entre lo escrito y la práctica.

\section{Conclusiones}

México, en específico Tenancingo tiene una gran riqueza cultural y natural que puede ser utilizada para que las personas la conozcan y la visiten. Sin embargo, hasta el momento no existe una visión estructurada para que los recursos sean usados de forma moderada y no desaparezcan.

Debe haber políticas públicas sobre cuidado de los recursos naturales y culturales y su mantenimiento. Así como programas educativos para la población en general sobre cuidado del medio ambiente; porque los recursos nos dan una vida saludable y se puede obtener una remuneración económica por su conservación.

Los tres sectores: el público, el privado y el social, quieren sacar una mejor ganancia cada uno por su lado, y lo único que se genera con esa actitud es no avanzar en la promoción de los recursos y obvio no se tiene un beneficio de ellos. En el momento que cada sector entienda su participación, en la promoción de los recursos naturales y culturales, habrá desarrollo local, de lo contrario se seguirán integrando estrategias, sin lograr nada. Tal vez exista crecimiento, pero no el desarrollo integral del municipio. 


\section{Referencias bibliografías}

Aparicio, M. (2009). Modelo de gestión por proyectos para el desarrollo local (TTT). XIII Congreso Internacional de Ingeniería de Proyectos. España: Universidad Politécnica de Madrid.

Boucher, F. et al, (2011). Guía Metodológica, para la Activación de Sistemas Agroalimentarios Localizados (SIAL). México: IICA, CIRAD, REDSIAL, México IICA. Cárdenas, N. (2002). "El desarrollo local su conceptualización y procesos". PROVINCIA. Universidad de los Andes Mérida, Venezuela, № 8, pág. 53-76.

Estadística. (2013). Estadística básica municipal Tenancingo. México: Gobierno del Estado de México.

Flores, C; Zizumbo, L; Cruz, G; Vargas, E. E. (2014). "Economía social, comunalidad: orientación teórica para el turismo rural, como alternativa de desarrollo". Revista Mexicana de Ciencias Agrícolas, núm. 9, pág. 1645-1658.

Gaceta Municipal. (2013). Plan de desarrollo municipal, 2013-2015. México: Ayuntamiento de Tenancingo. Gobierno del Estado de México. REPP-SPM-58-123.

Hernández, R; Fernández, C y P. Baptista (2010). Metodología de la investigación. México: Mc. Graw-Hill.

INEGI. (2013). Estadística Básica Municipal. México: Gobierno del Estado de México. INEGI. (2017). Anuario estadístico y geográfico de México. México: INEGI.

Juárez, G. (2013). "Revisión del concepto de desarrollo local desde una perspectiva territorial”. Revista Lider. Vol. 23, pág. 9-28.

Martínez, A; Escalona, M. (2012). "La gestión turística municipal en el Archipielago de Chiloé". Estudios y Perspectivas en Turismo, Centro de Investigaciones y Estudios Turísticos Buenos Aires, Argentina, vol. 21, núm. 3, pág. 621-645.

Muchnik, J. (2006). "Identidad territorial de los alimentos: alimentar el cuerpo humano y el cuerpo social”. En: De Álvarez, A., Bouher, F., Cervantes, F., Espinoza, A., Muchnik, J., y Requier Desjardins, D. (Cords.). Agroindustria Rural y Territorio. México: Universidad Autónoma del Estado de México.

OMT. La Organización Mundial del Turismo. (Madrid, España). [online]. 2018, [citado 5 abril 2018]. Disponible en la world wide web: http://www2.unwto.org/node/50285.

OMT. El turismo internacional hacia otro año de crecimiento. [online]. 2017, [citado 5 abril 2018]. Disponible en la world wide web: http://media.unwto.org/es/pressrelease/2017-11-07/el-turismo-internacional-hacia-otro-ano-de-crecimiento.

OMT. (1999). Agenda para Planificadores Locales: turismo sostenible y gestión municipal. Edición para América Latina y El Caribe. Madrid, España: OMT.

Ornés, S. (2009). "La gestión pública del turismo en el distrito metropolitano de Caracas. Fortalezas, debilidades y nuevos desafíos". Gestión turística, Universidad Austral de Chile Valdivia, Chile. $\mathrm{N}^{\circ} 12$, pág. 85-108.

Pérez, B. (S/A). Desarrollo Local: Presente y Futuro. Sevilla, España: Facultad de 
Ciencias Económicas y Empresariales. Departamento de Economía Aplicada III, de la Universidad de Sevilla.

PND. (2013-2018). Plan Nacional de Desarrollo 2013-2018. México: Gobierno de la República.

Rodríguez, J. (2015). "Gestión local del turismo: el municipio y su rol en el desarrollo turístico en Chile". Gestión Turística, Universidad Austral de Chile Valdivia, Chile. Núm. 23, pág. 34-56

SECTUR. Informe de Avance y Resultados del Programa Sectorial de Turismo. (lugar). [online]. 2017. [citado 10 abril 2018]. Disponible en la world wide web: https://www. gob.mx/sectur/\#200.

SECTUR. (2018). Ranking Mundial del Turismo Internacional. (lugar). [online]. 2018. [citado 15 abril 2018]. Disponible en la world wide web: http://www.datatur.sectur.gob. $\mathrm{mx} /$ SitePages/RankingOMT.aspx.

Varela, S. El obispo, baluarte gastronómico de Tenancingo 16 y 17 de Julio, la $8^{\text {a }}$ Feria del Obispo. (México). [online]. 2011. [citado 15 mayo 2018]. Disponible en la world wide web: https://alcommuni.wordpress.com/2011/07/07/\%E2\%80\%9Cel-obispo-baluartegastronomico-de-tenancingo\%E2\%80\%9D-17-y-18-de-julio-la-8\%C2\%AA-feria-delobispo/. Boletín A-32/11.

Vázquez, G. V. (2003). "La gestión ambiental con perspectiva de género: el manejo integrado de ecosistemas y la participación comunitaria". Gestión y políticas públicas. Vol. XII, núm. 2, ii semestre, pág. 291-322.

Velasco, G. M. (2009). "Gestión turística del patrimonio cultural: enfoques para un desarrollo sostenible del turismo cultural". Cuadernos de turismo. Núm. 23, pág. 237257.

Venturini, E. J. (2015). “Ambiente, sustentabilidad y turismo. La gestión ambiental como perspectiva para el desarrollo turístico sustentable". Revista Pensum, volumen 1, pág. $8-23$. 